\title{
Quantization of Electromagnetic Modes in a Hyperbolic Negative-Index Layered Superconductor Slab
}

\author{
S. Cortés-López* And F. PÉrez-Rodríguez \\ Instituto de Física, Benemérita Universidad Autónoma de Puebla, \\ Apartado Postal J-48, Puebla, Pue. 72570, Mexico
}

\begin{abstract}
We theoretically study the infrared reflectivity and transmissivity of a high-temperature layered superconductor slab. Both infrared spectra exhibit very narrow Fabry-Perot resonances associated with the quantization of the wave vector of the TM electromagnetic modes. The resonances are observed in a pass band where the refractive index of the layered superconductor is negative. The pass band of negative dispersion is above the Josephson plasma frequency which appears in the expression for the effective permittivity component, corresponding to the direction perpendicular to the layers. It was found that the Fabry-Perot resonances undergo a blue shift as the slab thickness or the angle of incidence are increased. Moreover, the quantized electromagnetic modes turn out to be quasi-longitudinal because of the strong anisotropy of the infrared dielectric response of the layered superconductor.
\end{abstract}

DOI: 10.12693/APhysPolA.130.641

PACS/topics: 74.25.Gz, 74.25.N-, 74.78.Fk, 78.67.Pt

\section{Introduction}

Metamaterials with negative refractive index can be constructed by alternating metallic and dielectric layers (see, e.g., [1-3]), forming thus a periodic heterostructure. Such kind of metamaterials conform, in fact, a one-dimensional (1D) photonic crystal (PC), also known as superlattice.

When the wavelength of the incident electromagnetic field is much larger than the PC period (regime of long wavelengths), the PC optical properties can be described by using an effective permittivity tensor, which in general is frequency dependent. Besides, if the principal components of this effective permittivity tensor are of different sign, the anisotropic medium is known as a hyperbolic metamaterial because it can support negative refraction while its dispersion relation represents a hyperbole equation in the wave vector space $\boldsymbol{k}$. The advantage of hyperbolic metamaterials over double-negative metamaterials, having simultaneously negative permittivity and permeability, is the possibility to use metallic inclusions, in the unit cell, of simple forms without having negative permeability.

All metamaterials above described are made of metal and dielectric (conventional metamaterials). In microwave and terahertz $(\mathrm{THz})$ frequency ranges they produce energy losses mainly associated to the metallic component and with the resonant generation of their magnetic effective response. Therefore, substantial losses are one of the key limitations of conventional metamaterials. A way to avoid such a disadvantage has been developed in recent years and consists in the substitution of the

\footnotetext{
*corresponding author; e-mail: scortesl@ifuap.buap.mx
}

metallic component in the metamaterial for another one, but made of superconductor. As a result of using superconductors, the energy losses can be reduced in the $\mathrm{THz}$ range.

As is shown in Ref. [4], layered cuprate superconductors and artificial superconducting-insulator systems are strongly anisotropic metamaterials, which can posseses negative index of refraction in a wide frequency range for arbitrary incident angles. In such anisotropic metamaterials, the permittivity tensor components, along and transverse to the superconducting layers have different signs above the infrared Josephson plasma frequency. A detailed analysis of the dispersion curves for waveguide and surface Josephson plasma waves in a slab of layered superconductor placed between two identical dielectrics is reported in Ref. [5]. There, it was shown that the eigenmodes can be resonantly excited in the slab by means of the attenuated-total-reflection method.

In the present work, we theoretically investigate infrared spectra of a high-temperature layered superconductor slab. We will use the macroscopic permittivity tensor, obtained in Ref. [4], for calculating the electromagnetic field inside the superconductor slab and, then, the reflectivity and transmissivity for both TM- and TEpolarization geometries. Specifically, we will study the effect of the high anisotropy in the high-temperature superconductor dielectric response on such spectra. Besides, the effects of the slab thickness and angle of the incident light upon infrared spectra will be analyzed. In Sect. 2, general formulae of the permittivity tensor and TM transmissivity for the layered-superconductor slab are presented. The numerically-calculated transmissivity spectra for a $\mathrm{Bi}_{2} \mathrm{Sr}_{2} \mathrm{CaCu}_{2} \mathrm{O}_{8+\delta}$ slab are shown and commented in Sect. 3. Finally, there is a section of Conclusions (Sect. 4). 


\section{Theoretical formalism}

Let us consider a high-temperature layered superconducting slab, being in vacuum and occupying the space $0 \leq z \leq d$ (see Fig. 1). The superconducting layers are assumed to be parallel to the $x-y$ plane. A monochromatic electromagnetic plane wave with $p$-polarization is incident on the superconductor-slab surface at $z=0$. According to the system geometry, the magnetic field of the wave can be expressed as

$$
\boldsymbol{H}_{i}=\left(0, H_{i}, 0\right) \mathrm{e}^{\mathrm{i}\left(k_{x} x+k_{z} z-\omega t\right)}, \quad z \leq 0,
$$

where $\omega$ is the frequency, $k_{x}=k \sin \theta$ and $k_{z}=k \cos \theta$ are the components of the incident wave vector $\boldsymbol{k}_{i}, k=\omega / c$, $c$ is the light velocity in vacuum, and $\theta$ is the incidence angle. The magnetic field of the reflected wave is given by

$$
\boldsymbol{H}_{r}=\left(0, H_{r}, 0\right) \mathrm{e}^{\mathrm{i}\left(k_{x} x-k_{z} z-\omega t\right)}, \quad z \leq 0 .
$$

At $z>d$ the magnetic component of the transmitted electromagnetic wave can be written as

$$
\boldsymbol{H}_{t}=\left(0, H_{t}, 0\right) \mathrm{e}^{\mathrm{i}\left[k_{x} x+k_{z}(z-d)-\omega t\right]}, \quad z \geq d .
$$

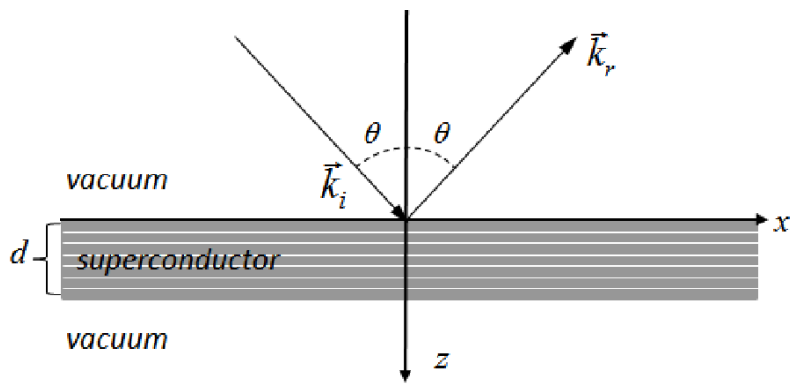

Fig. 1. Scheme of a high-temperature layered superconducting slab. $\boldsymbol{k}_{i}$ and $\boldsymbol{k}_{r}$ are respectively the wave vectors of the incident and reflected light.

In the continuous limit, i.e. when the characteristic wavelength is much larger than the period of the hightemperature layered superconductor along the growth direction ( $z$ axis), the superconductor behaves as a uniaxial crystal with principal values of its permittivity tensor $\stackrel{\leftrightarrow}{\epsilon}$ given by [4]:

$$
\epsilon_{x}=\epsilon_{y}=\epsilon\left(1-\frac{\gamma^{2} \omega_{p}^{2}}{\omega^{2}}\right), \quad \epsilon_{z}=\epsilon\left(1-\frac{\omega_{p}^{2}}{\omega^{2}}\right),
$$

where $\omega_{p}=c /\left(\lambda_{\perp} \sqrt{\epsilon}\right)$ is the Josephson plasma frequency, $\gamma=\lambda_{\perp} / \lambda_{\|}$is the anisotropic parameter given by the ratio between the transverse and perpendicular magnetic penetration depths.

Using Eqs. (4) and the Maxwell equations, the magnetic field of the electromagnetic wave inside the superconductor slab can be written as

$$
\boldsymbol{H}_{s}=\left(0, H_{s}(z), 0\right) \mathrm{e}^{\mathrm{i}\left(k_{x} x-\omega t\right)}, \quad 0 \leq z \leq d,
$$

where

$$
H_{s}(z)=H_{1} \mathrm{e}^{\mathrm{i} k_{z}^{(s)} z}+H_{2} \mathrm{e}^{-\mathrm{i} k_{z}^{(s)} z} .
$$

The wave-vector component $k_{z}^{(s)}$ is given by the formula

$$
k_{z}^{(s)}=\sqrt{\epsilon_{x}\left(\frac{\omega^{2}}{c^{2}}-\frac{k_{x}^{2}}{\epsilon_{z}}\right)} .
$$

The electric field $\boldsymbol{E}$ for the electromagnetic waves inside the anisotropic superconductor and in vacuum is calculated by employing Eqs. (1)-(6) and the AmpereMaxwell law $\left(\boldsymbol{E}=(\mathrm{i} c / \omega) \stackrel{\leftrightarrow}{\epsilon}^{-1} \nabla \times \boldsymbol{H}\right)$. In order to determine the amplitudes $H_{r}, H_{t}, H_{1}$, and $H_{2}$ in terms of the amplitude of the incident wave $\left(H_{i}\right)$, we should apply the Maxwell boundary conditions, namely the continuity of the tangential components of the magnetic and electric field at the surfaces $z=0$ and $z=d$. Afterwards, the $p$-polarization reflectivity $\left(R_{p}=\left|H_{r} / H_{i}\right|\right)$ and transmissivity $\left(T_{p}=\left|H_{t} / H_{i}\right|\right)$ are straightforwardly calculated. Analytical expressions for $R_{p}$ and $T_{p}$ are presented in Refs. [2, 6, 7]. The TM transmissivity is [2]:

$$
T_{p}=\frac{1}{\left|\cos \left(k_{z}^{(s)} d\right)-\frac{\mathrm{i}}{2}\left(\frac{k_{z} \epsilon_{x}}{k_{z}^{(s)}}+\frac{k_{z}^{(s)}}{k_{z} \epsilon_{x}}\right) \sin \left(k_{z}^{(s)} d\right)\right|^{2}} .
$$

\section{Numerical results and discussion}

In this section we will apply the theoretical formalism described above. In part (a) of Fig. 2 we present the dispersion relation $\omega\left(k_{z}^{(s)}\right)$ for transverse-magnetic (TM) modes in $\mathrm{Bi}_{2} \mathrm{Sr}_{2} \mathrm{CaCu}_{2} \mathrm{O}_{8+\delta}$ superconductor at $\theta=45^{\circ}$, which was calculated by using the formulae (4) for the principal values of the average permittivity tensor and Eq. (7). In the calculation, we have used the parameters for $\mathrm{Bi}_{2} \mathrm{Sr}_{2} \mathrm{CaCu}_{2} \mathrm{O}_{8+\delta}[4]: \epsilon=12.0, \omega_{p}=10^{12} \mathrm{~s}^{-1}$ and $\gamma=500$. In part (b) we exhibit the spectrum of the transmissivity $T_{p}$ for a $\mathrm{Bi}_{2} \mathrm{Sr}_{2} \mathrm{CaCu}_{2} \mathrm{O}_{8+\delta}$ superconductor slab of thickness $d=\delta$, where $\delta=c /\left(\gamma \omega_{p} \sqrt{\epsilon}\right)$. Here, we have neglected the energy losses in the hightemperature superconductor.

As is seen in Fig. 2a, at frequencies $\omega<\omega_{p}$ the wave vector $k_{z}^{(s)}(7)$ is purely imaginary because both $\epsilon_{x}(\omega)$ and $\epsilon_{z}(\omega)$ (4) are negative. Above the Josephson plasma frequency $\left(\omega>\omega_{p}\right)$ the wave vector $k_{z}^{(s)}$ is real and negative, since $\epsilon_{z}$ has changed its sign, and the refraction index of the superconductor turns out to be negative.

It is surprising that the transmission (see Fig. 2b) in the pass band of negative dispersion is zero in almost the whole band except at certain frequencies, where sharp resonances are observed. This is a direct consequence of the high dielectric contrast between the superconductor and vacuum. Indeed, in this case the quantity between parenthesis in the formula (8) is rather large and, therefore, $T_{p}$ is very small. However, at frequencies where $\sin \left(k_{z}^{(s)} d\right)$ in Eq. (8) vanishes, the transmissivity for TM modes is equal to unity. The later occurs when the Fabry-Perot resonance condition is fulfilled

$$
\left|k_{z}^{(s)}\right| d=n \pi \quad n=1,2, \ldots
$$

The fact that the resonance frequencies in the infrared transmissivity spectrum $T_{p}(\omega)$ correspond to values of the quantized wave vector $k_{z}^{(s)}$ can be confirmed by comparing the dispersion relation $k_{z}^{(s)}(\omega)$ (part (a) of Fig. 2) and the transmissivity (part (b) therein). 

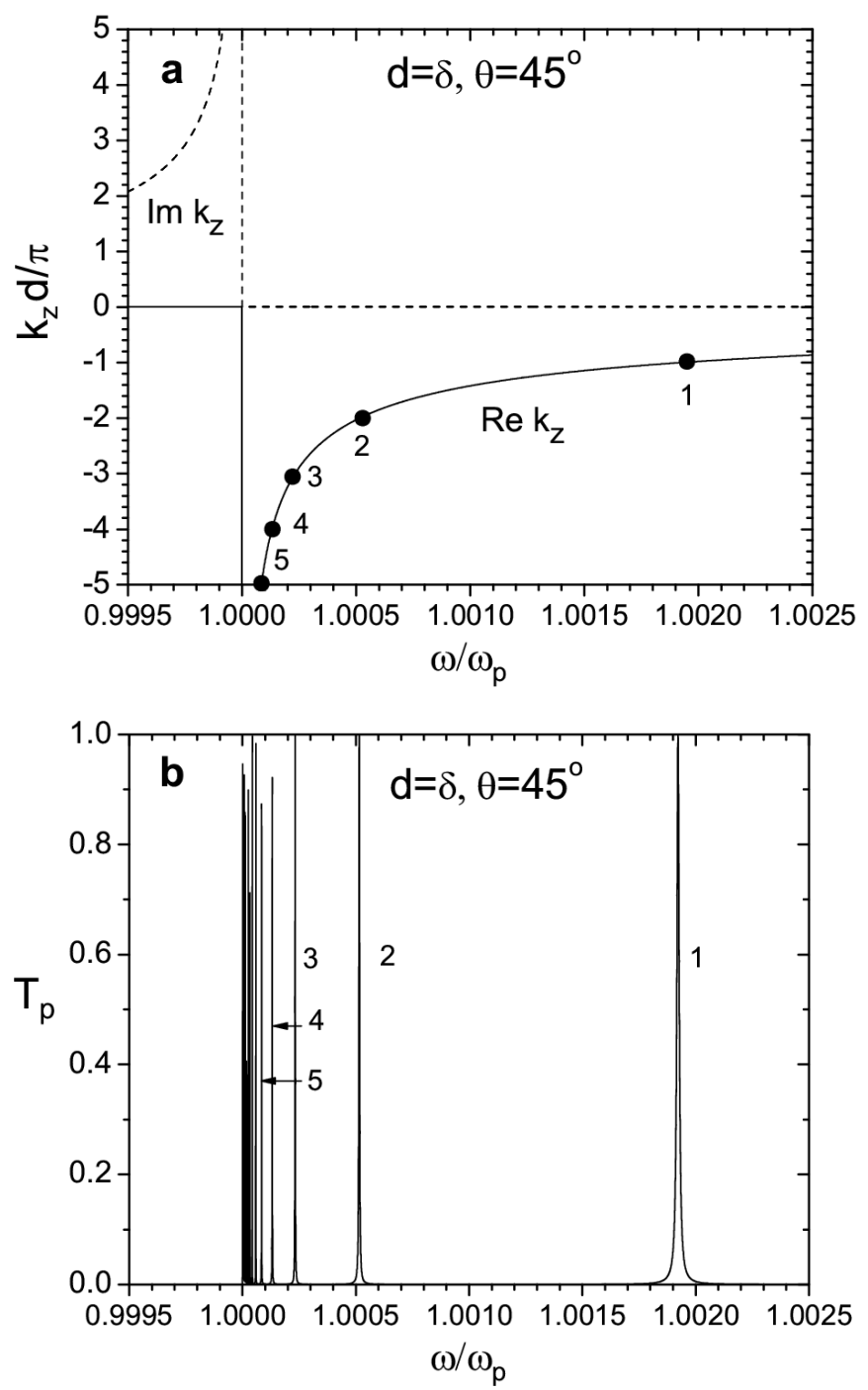

Fig. 2. (a) Dispersion relation $\omega\left(k_{z}^{(s)}\right)$ for TM modes in $\mathrm{Bi}_{2} \mathrm{Sr}_{2} \mathrm{CaCu}_{2} \mathrm{O}_{8+\delta}$ superconductor at $\theta=45^{\circ}$. (b) Transmissivity spectrum $T_{p}$ for the high-temperature superconducting slab of thickness $d=\delta$. Numbers $1,2, \ldots 5$ indicate the value of $n$ at frequencies of the first five Fabry-Perot resonances (Eq. (9)).

It should be mentioned that the transmissivity FabryPerot resonances undergo a blue shift, with increasing the thickness $d$, due to the negative dispersion $\omega\left(k_{z}^{(s)}\right)$ (Fig. 2a) for the high-temperature superconductor slab in contrast to the red shift observed in the case of slabs having positive refractive index. Indeed, according to Eq. (9) (Fig. 2a), as $d$ is increased, $\left|k_{z}^{(s)}\right|$ decreases and, consequently, the frequencies of the Fabry-Perot resonances are shifted to the blue. The resonances, associated to quantized modes, are also shifted to higher frequencies if the angle of incidence $\theta$ is increased. The blue shift owing to the increase of $\theta$ is clearly observed in Fig. 3, where transmissivity spectra for TM modes at the angles $\theta=45^{\circ}$ (solid line) and $\theta=75^{\circ}$ (dotted line) are exhibited.

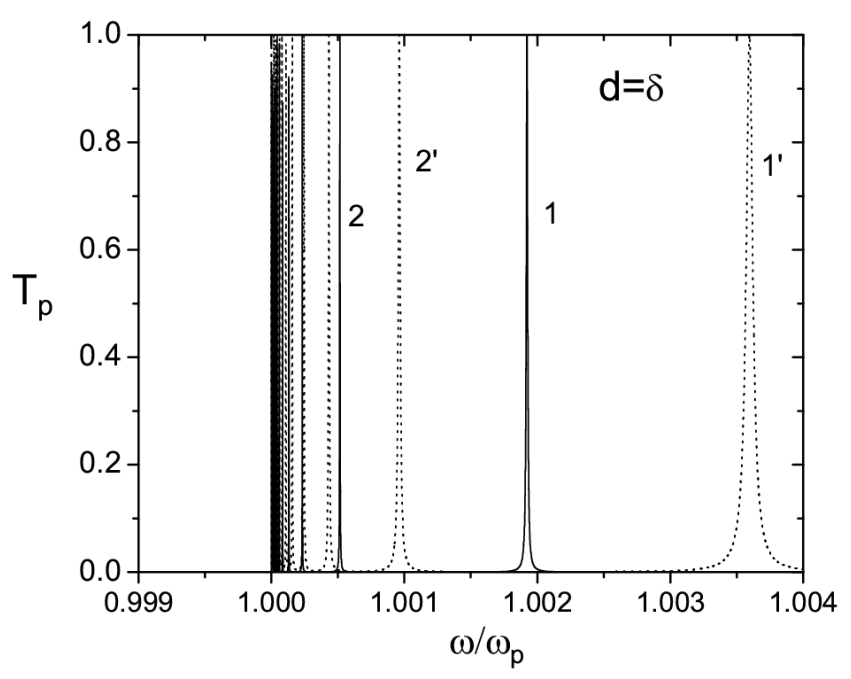

Fig. 3. Transmissivity spectra $T_{p}$ for the hightemperature superconducting slab of thickness $d=\delta$ at the angles of incidence $\theta=45^{\circ}$ (solid line) and $75^{\circ}$ (dotted line).

The $z$ component $\left(k_{z}^{(s)}\right)$ of the wave vector for the propagating TM modes inside the uniaxial superconductor crystal (see Eq. (5)) is much larger than the $x$ component $k_{x}$ because of the high anisotropy of its dielectric response $\left(\left|-\epsilon_{x} / \epsilon_{z}\right| \gg 1\right.$ at $\left.\omega \gtrsim \omega_{p}\right)$. Certainly, as follows from Eq. (7):

$$
\frac{k_{z}}{k_{x}} \approx \sqrt{\frac{-\epsilon_{x}}{\epsilon_{z}}} \gg 1 .
$$

On the other hand, the wave vector of the electromagnetic TM waves is perpendicular to the displacement vector $\boldsymbol{D}$ because of the Maxwell equation $\nabla \cdot \boldsymbol{D}=0$. Hence, the magnitude of the $z$ component $\left(E_{z}\right)$ of the electric field turns out to be rather large in comparison with that of the $x$ component $\left(E_{x}\right)$ at $\omega \gtrsim \omega_{p}$ :

$$
\left|E_{z}\right|=\left|\frac{-k_{x} \epsilon_{x}}{k_{z}^{(s)} \epsilon_{z}} E_{x}\right| \approx\left|\sqrt{\frac{-\epsilon_{x}}{\epsilon_{z}}} E_{x}\right| \gg\left|E_{x}\right| .
$$

According to Eqs. (10) and (11), the propagating TM modes in the high-temperature superconductor are quasilongitudinal since their wave vector and electric field are almost parallel to the $z$ axis.

Finally, we should comment that the infrared spectra of reflectivity $\left(R_{s}\right)$ and transmissivity $\left(T_{s}\right)$ for $s$ polarization incident light were also calculated. Such spectra are completely determined by the permittivity component $\epsilon_{y}$ which is negative in $\mathrm{THz}$ range. As a result, the transverse-electric (TE) modes are evanescent at $\omega \sim \omega_{p}$ and $R_{s}\left(T_{s}\right)$ is almost equal to one (zero).

\section{Conclusions}

Optical spectra (reflectivity and transmissivity) of a high-temperature layered superconductor slab were calculated. Such a slab behaves as a hyperbolic metamaterial with negative refraction index just above the infrared Josephson plasma frequency $\omega_{p}$ appearing in the 
expression for the effective permittivity component, $\epsilon_{z}$ in Eq. (4). The calculated $p$-polarization transmissivity exhibits narrow Fabry-Perot resonances corresponding to quantized electromagnetic modes inside the superconductor slab. It was established that the Fabry-Perot resonances undergo a blue shift if the slab thickness or the angle of the incident light are increased. The resonance shift, associated with the increase of the slab thickness, is due to the negative dispersion of the pass band for the TM modes. Besides, because of the high anisotropy of the superconductor dielectric response $\left|\epsilon_{x} / \epsilon_{z}\right| \gg 1$, the quantized TM modes (at $\omega \geq \omega_{p}$ ) turn out to be quasilongitudinal since their wave vector and electric field are almost parallel to the $z$ axis.

\section{Acknowledgments}

This work was partially supported by SEP-CONACYT (grant 183673), VIEP-BUAP, and PIFCA-2015.

\section{References}

[1] V.A. Podolskiy, E.E. Narimanov, Phys. Rev. B 71, 201101(R) (2005).

[2] B. Wood, J.B. Pendry, D.P. Tsai, Phys. Rev. B 74, 115116 (2006).

[3] B. Zenteno-Mateo, V. Cerdán-Ramírez, B. FloresDesirena, M.P. Sampedro, E. Juárez-Ruiz, F. PérezRodríguez, Progr. Electromagn. Res. Lett. 22, 165 (2011).

[4] A.L. Rakhmanov, V.A. Yampol'skii, J.A. Fan, F. Capasso, F. Nori, Phys. Rev. B 81, 075101 (2010).

[5] T.M. Slipchenko, D.V. Kadygrob, D. Bogdanis, V.A. Yampol'skii, A.A. Krokhin, Phys. Rev. B 84, 224512 (2011).

[6] O.E. Piro, Phys. Rev. B 36, 3427 (1987).

[7] A. Silva-Catillo, F. Pérez-Rodríguez, Phys. Status Solidi B 219, 215 (2000). 